\title{
Some strong laws of large numbers for weighted sums of asymptotically almost negatively associated random variables
}

\section{Xiaofeng Tang}

"Correspondence:

tang_xiao_feng2012@126.com School of Mathematics and Computing Science, Fuyang Teachers College, Fuyang, 236041, P.R. China

\begin{abstract}
In the paper, we study the strong law of large numbers for general weighted sums of asymptotically almost negatively associated random variables (AANA, in short) with non-identical distribution. As an application, the Marcinkiewicz strong law of large numbers for AANA random variables is obtained. In addition, we present some sufficient conditions to prove the strong law of large numbers for weighted sums of AANA random variable. Our results generalize the corresponding ones (sufficient conditions) for independent random variables.

MSC: $60 \mathrm{~F} 15$

Keywords: asymptotically almost negatively associated random variables; Marcinkiewicz strong law of large numbers; weighted sums; the three series theorem
\end{abstract}

\section{Introduction}

Throughout the paper, let $\left\{X, X_{n}, n \geq 1\right\}$ be a sequence of random variables defined on a fixed probability space $(\Omega, \mathcal{F}, P)$. $C$ denotes a positive constant not depending on $n$, which may be different in various places. Let $\lceil x\rceil$ denote the integer part of $x$ and $I(A)$ be the indicator function of the set $A$.

Recently, Jajte [1] studied the strong law of large numbers for general weighted sums of independent and identically distributed random variables. The main result of Jajte [1] is as follows.

Theorem 1.1 Let $\left\{X_{n}, n \geq 1\right\}$ be a sequence of independent and identically distributed random variables. Let $g(\cdot)$ be a positive, increasing function and $h(\cdot)$ be a positive function such that $\phi(y) \equiv g(y) h(y)$ satisfies the following conditions.

(i) For some $d \geq 0, \phi(\cdot)$ is strictly increasing on $[d, \infty)$ with range $[0, \infty)$.

(ii) There exist $C$ and a positive integer $k_{0}$ such that $\phi(y+1) / \phi(y) \leq C$ for all $y \geq k_{0}$.

(iii) There exist constants $a$ and $b$ such that for all $s>d$,

$$
\phi^{2}(s) \int_{s}^{\infty} \frac{1}{\phi^{2}(x)} d x \leq a s+b
$$

Then the following two conditions are equivalent:

(1) $E\left[\phi^{-1}\left(\left|X_{1}\right|\right)\right]<\infty$, 
(2) $\frac{1}{g(n)} \sum_{i=1}^{n} \frac{X_{i}-m_{i}}{h(i)} \rightarrow 0$ a.s. as $n \rightarrow \infty$, where $m_{i}=E X_{i} I\left(\left|X_{i}\right| \leq \phi(i)\right)$ and $\phi^{-1}$ is the inverse of a function $\phi$.

Inspired by Jajte [1], Jing and Liang [2] extended the result of Jajte [1] for independent and identically distributed random variables to the case of negatively associated random variables with identical distribution. Meng and Lin [3] and Wang [4] extended Theorem 1.1 to the case of $\tilde{\rho}$-mixing random variables and non-identically distributed negatively associated random variables, respectively. Sung [5] gave some sufficient conditions to prove the strong law of large numbers for weighted sums of random variables. The main purpose of the paper is to generalize the result of Theorem 1.1 to the case of asymptotically almost negatively associated random variables, which contains independent random variables and negatively associated (NA, in short) random variables as special cases. In addition, we present some sufficient conditions to prove the strong law of large numbers for weighted sums of asymptotically almost negatively associated random variables.

The concept of asymptotically almost negatively associated random variables is as follows.

Definition 1.1 A sequence $\left\{X_{n}, n \geq 1\right\}$ of random variables is called asymptotically almost negatively associated (AANA, in short) if there exists a nonnegative sequence $q(n) \rightarrow 0$ as $n \rightarrow \infty$ such that

$$
\operatorname{Cov}\left(f\left(X_{n}\right), g\left(X_{n+1}, X_{n+2}, \ldots, X_{n+k}\right)\right) \leq q(n)\left[\operatorname{Var}\left(f\left(X_{n}\right)\right) \operatorname{Var}\left(g\left(X_{n+1}, X_{n+2}, \ldots, X_{n+k}\right)\right)\right]^{1 / 2}
$$

for all $n, k \geq 1$ and for all coordinatewise nondecreasing continuous functions $f$ and $g$ whenever the variances exist.

The family of AANA sequence contains NA (in particular, independent) sequences (with $q(n)=0, n \geq 1$ ) and some more sequences of random variables which are not much deviated from being negatively associated. For more details about NA random variables, one can refer to Joag-Dev and Proschan [6], and so forth. An example of an AANA sequence which is not NA was constructed by Chandra and Ghosal [7].

Since the concept of AANA sequence was introduced by Chandra and Ghosal [7], many applications have been found. For example, Chandra and Ghosal [7] derived the Kolmogorov type inequality and the strong law of large numbers of Marcinkiewicz-Zygmund; Chandra and Ghosal [8] obtained the almost sure convergence of weighted averages; Wang et al. [9] established the law of the iterated logarithm for product sums; Ko et al. [10] studied the Hájek-Rényi type inequality; Yuan and An [11] established some Rosenthal type inequalities for maximum partial sums of AANA sequence; Yuan and Wu [12] studied the limiting behavior of the maximum of the partial sum for asymptotically negatively associated random variables under residual Cesàro alpha-integrability assumption; Wang et al. [13] obtained some strong growth rate and the integrability of supremum for the partial sums of AANA random variables; Yuan and An [14] established some laws of large numbers for Cesàro alpha-integrable random variables under dependence condition AANA or AQSI; Wang et al. [15] studied the complete convergence for weighted sums of arrays of rowwise AANA random variables; and Yang et al. [16] investigated the complete convergence of moving average process for AANA sequence, and so forth. 
The structure of this paper is as follows. Some important lemmas are presented in Section 2. Main results and their proofs are provided in Section 3 and Section 4, respectively.

\section{Preliminaries}

In this section, we will present some important lemmas which will be used to prove the main results of the paper. The first one is a basic property of AANA random variables, which was given by Yuan and An [11].

Lemma 2.1 (cf. Yuan and An [11]) Let $\left\{X_{n}, n \geq 1\right\}$ be a sequence of AANA random variables with mixing coefficients $\{q(n), n \geq 1\}, f_{1}, f_{2}, \ldots$ be all nondecreasing (or nonincreasing) continuous functions, then $\left\{f_{n}\left(X_{n}\right), n \geq 1\right\}$ is still a sequence of $A A N A$ random variables with mixing coefficients $\{q(n), n \geq 1\}$.

The next one, the Khintchine-Kolmogorov type convergence theorem for AANA random variables proved by Wang et al. [17], will play an essential role in proving the main results of the paper.

Lemma 2.2 (cf. Wang et al. [17]) Let $\left\{X_{n}, n \geq 1\right\}$ be a sequence of AANA random variables with mixing coefficients $\{q(n), n \geq 1\}$ and $\sum_{n=1}^{\infty} q^{2}(n)<\infty$. Assume that $\sum_{n=1}^{\infty} \operatorname{Var}\left(X_{n}\right)<\infty$, then $\sum_{n=1}^{\infty}\left(X_{n}-E X_{n}\right)$ converges a.s.

By Lemma 2.2 and the standard method, we can easily get the following three series theorem for AANA random variables. The proof is easy, so we omit the details.

Lemma 2.3 Let $\left\{X_{n}, n \geq 1\right\}$ be a sequence of AANA random variables with mixing coefficients $\{q(n), n \geq 1\}$ and $\sum_{n=1}^{\infty} q^{2}(n)<\infty$. Denote $X_{n}^{(c)}=-c I\left(X_{n}<-c\right)+X_{n} I\left(\left|X_{n}\right| \leq c\right)+$ $c I\left(X_{n}>c\right)$, where $c$ is a positive constant. If the following three conditions are satisfied:

(i) $\sum_{n=1}^{\infty} P\left(\left|X_{n}\right|>c\right)<\infty$;

(ii) $\sum_{n=1}^{\infty} E X_{n}^{(c)}$ converges;

(iii) $\sum_{n=1}^{\infty} \operatorname{Var} X_{n}^{(c)}<\infty$,

then $\sum_{n=1}^{\infty} X_{n}$ converges a.s.

The following concept of stochastic domination will be used in this paper.

Definition 2.1 A sequence $\left\{X_{n}, n \geq 1\right\}$ of random variables is said to be stochastically dominated by a random variable $X$ if there exists a positive constant $C$ such that

$$
P\left(\left|X_{n}\right|>x\right) \leq C P(|X|>x)
$$

for all $x \geq 0$ and $n \geq 1$.

By the definition of stochastic domination and integration by parts, we can get the following basic inequalities. The proof is standard, so we omit it.

Lemma 2.4 Let $\left\{X_{n}, n \geq 1\right\}$ be a sequence of random variables which is stochastically dominated by a random variable $X$. For any $\alpha>0$ and $b>0$, the following two statements 
hold:

$$
\begin{aligned}
& E\left|X_{n}\right|^{\alpha} I\left(\left|X_{n}\right| \leq b\right) \leq C_{1}\left[E|X|^{\alpha} I(|X| \leq b)+b^{\alpha} P(|X|>b)\right], \\
& E\left|X_{n}\right|^{\alpha} I\left(\left|X_{n}\right|>b\right) \leq C_{2} E|X|^{\alpha} I(|X|>b),
\end{aligned}
$$

where $C_{1}$ and $C_{2}$ are positive constants. Consequently, $E\left|X_{n}\right|^{\alpha} \leq C E|X|^{\alpha}$.

\section{Main results}

Hypothesis A Let $f(x)$ and $g(x)$ be real positive functions defined on the same domain $(0, \infty)$ and $\varphi(x)=f(x) g(x)(\varphi(0)=0) . \varphi(x)$ is strictly increasing on $[0, \infty), \lim _{x \rightarrow \infty} \varphi(x)=\infty$, and its range is $[0, \infty)$.

Hypothesis B There exist constants $a, b \in \mathbb{R}$ such that for every $t \in \mathbb{R}$,

$$
t^{2} \int_{\varphi^{-1}(|t|)}^{\infty} \frac{1}{\varphi^{2}(x)} d x \leq a \varphi^{-1}(|t|)+b .
$$

Throughout the paper, let $\left\{X_{n}, n \geq 1\right\}$ be a sequence of AANA random variables with mixing coefficients $\{q(n), n \geq 1\}$ and $\sum_{n=1}^{\infty} q^{2}(n)<\infty$, which is stochastically dominated by a random variable $X$. Denote $m_{n}=E X_{n} I\left(\left|X_{n}\right| \leq \varphi(n)\right)$ for each $n \geq 1$. Based on Hypothesis A and Hypothesis B, we will establish the strong law of large numbers for the weighted sums of AANA random variables. Our main results are as follows.

Theorem 3.1 Let $f(x), g(x)$ and $\varphi(x)$ be functions satisfying the conditions of Hypothesis A and Hypothesis B. If $E\left[\varphi^{-1}(|X|)\right]<\infty$, then

$$
\sum_{n=1}^{\infty} \frac{X_{n}-m_{n}}{\varphi(n)} \quad \text { converges a.s. }
$$

If we further assume that $f(x)$ is increasing on its domain and $\lim _{x \rightarrow \infty} f(x)=\infty$, then $\frac{1}{f(n)} \sum_{i=1}^{n} \frac{X_{i}-m_{i}}{g(i)} \rightarrow 0$ a.s. as $n \rightarrow \infty$.

Theorem 3.2 Let $f(x), g(x)$ and $\varphi(x)$ be functions satisfying the conditions of Hypothesis A and Hypothesis B. Assume further that $\varphi(x)$ satisfies the following conditions:

(i) If $\int_{r}^{\infty} \frac{1}{\varphi(x)} d x$ is finite, then $\int_{r}^{\infty} \frac{1}{\varphi(x)} d x \leq C_{1} r / \varphi(r)$, where $r \geq 1$ and $C_{1}>0$ are constants.

(ii) If $\int_{r}^{\infty} \frac{1}{\varphi(x)} d x$ does not exist or is infinite, then $x / \varphi(x)$ is nondecreasing and $\int_{1}^{t} \frac{1}{\varphi(x)} d x \leq C_{2} t / \varphi(t)$, where $r \geq 1, t \geq 1$ and $C_{2}>0$ are constants.

Suppose that $E\left[\varphi^{-1}(|X|)\right]<\infty$ and $E X_{i}=0$ when (ii) holds, then

$$
\sum_{n=1}^{\infty} \frac{X_{n}}{\varphi(n)} \quad \text { converges a.s. }
$$

If we further assume that $f(x)$ is increasing on its domain and $\lim _{x \rightarrow \infty} f(x)=\infty$, then $\frac{1}{f(n)} \sum_{i=1}^{n} \frac{X_{i}}{g(i)} \rightarrow 0$ a.s. as $n \rightarrow \infty$.

By Theorem 3.1 and Theorem 3.2, we can get the Marcinkiewicz strong law of large numbers for AANA random variables as follows. 
Corollary 3.1 Let $\left\{X_{n}, n \geq 1\right\}$ be a sequence of identically distributed AANA random variables with mixing coefficients $\{q(n), n \geq 1\}$ and $\sum_{n=1}^{\infty} q^{2}(n)<\infty$. If $E\left|X_{1}\right|^{p}<\infty$ for some $0<p<2$, then for some finite constant $a$,

$$
\frac{1}{n^{1 / p}} \sum_{i=1}^{n}\left(X_{i}-a\right) \rightarrow 0 \quad \text { a.s. as } n \rightarrow \infty .
$$

If $0<p<1$, then a can take an arbitrary real number. If $1 \leq p<2$, then $a=E X_{1}$.

The next two results are based on different conditions from Hypothesis B. The main idea is inspired by Sung [5]. Here we consider stochastic domination, not identical distribution.

Theorem 3.3 Let $f(x), g(x)$ and $\varphi(x)$ be functions satisfying the conditions of Hypothesis A. Assume further that the following conditions hold:

(i) $\sum_{n=1}^{\infty} P(|X|>\varphi(n))<\infty$;

(ii) $\sum_{n=1}^{\infty} \frac{1}{\varphi^{2}(n)} E X^{2} I(|X| \leq \varphi(n))<\infty$.

Then (3.2) holds true. If we further assume that $f(x)$ is increasing on its domain and $\lim _{x \rightarrow \infty} f(x)=\infty$, then $\frac{1}{f(n)} \sum_{i=1}^{n} \frac{X_{i}-m_{i}}{g(i)} \rightarrow 0$ a.s. as $n \rightarrow \infty$.

Theorem 3.4 Let $f(x), g(x)$ and $\varphi(x)$ be functions satisfying the conditions of Hypothesis A. Assume further that the following conditions hold:

(i) $\sum_{n=1}^{\infty} \frac{1}{\varphi(n)} E|X| I(|X|>\varphi(n))<\infty$;

(ii) $\sum_{n=1}^{\infty} \frac{1}{\varphi^{2}(n)} E X^{2} I(|X| \leq \varphi(n))<\infty$.

Then

$$
\sum_{n=1}^{\infty} \frac{X_{n}-E X_{n}}{\varphi(n)} \text { converges a.s. }
$$

If we further assume that $f(x)$ is increasing on its domain and $\lim _{x \rightarrow \infty} f(x)=\infty$, then $\frac{1}{f(n)} \sum_{i=1}^{n} \frac{X_{i}-E X_{i}}{g(i)} \rightarrow 0$ a.s. as $n \rightarrow \infty$.

Remark 3.1 The methods used in this paper are the Khintchine-Kolmogorov type convergence theorem and the three series theorem for AANA random variables, which are partially the same as that in Jajte [1] and Jing and Liang [2]. But here we consider some different conditions.

\section{Proofs of the main results}

Proof of Theorem 3.1 For every $n \geq 1$, denote

$$
Y_{n}=-\varphi(n) I\left(X_{n}<-\varphi(n)\right)+X_{n} I\left(|X|_{n} \leq \varphi(n)\right)+\varphi(n) I\left(X_{n}>\varphi(n)\right) .
$$

It is easily seen that

$$
\begin{aligned}
\sum_{n=1}^{\infty} P\left(X_{n} \neq Y_{n}\right) & =\sum_{n=1}^{\infty} P\left(\left|X_{n}\right|>\varphi(n)\right) \\
& \leq C \sum_{n=1}^{\infty} P(|X|>\varphi(n))
\end{aligned}
$$




$$
\begin{aligned}
& =C \sum_{n=1}^{\infty} P\left(\varphi^{-1}(|X|)>n\right) \\
& \leq C E\left[\varphi^{-1}(|X|)\right]<\infty,
\end{aligned}
$$

which together with the Borel-Cantelli lemma yields that

$$
\begin{aligned}
& P\left(\left(X_{n} \neq Y_{n}\right), \text { i.o. }\right)=P\left(X_{n} \neq Y_{n}, \text { i.o. }\right)=0, \\
& P\left(X_{n}=Y_{n}, \text { for } n \text { large enough }\right)=1 .
\end{aligned}
$$

Now, we consider the series $\sum_{n=1}^{\infty} \frac{E Y_{n}^{2}}{\varphi^{2}(n)}$. By $C_{r}$ 's inequality, Lemma 2.4 and (4.1), we can get that

$$
\begin{aligned}
\sum_{n=1}^{\infty} \frac{E Y_{n}^{2}}{\varphi^{2}(n)} & \leq C \sum_{n=1}^{\infty} \frac{1}{\varphi^{2}(n)}\left[E X_{n}^{2} I\left(\left|X_{n}\right| \leq \varphi(n)\right)+\varphi^{2}(n) P\left(\left|X_{n}\right|>\varphi(n)\right)\right] \\
& \leq C \sum_{n=1}^{\infty} \frac{E X^{2} I(|X| \leq \varphi(n))}{\varphi^{2}(n)}+C \sum_{n=1}^{\infty} P(|X|>\varphi(n)) \\
& \leq C E\left[\sum_{n=1}^{\infty} \frac{X^{2} I(|X| \leq \varphi(n))}{\varphi^{2}(n)}\right] .
\end{aligned}
$$

Since $E\left[\varphi^{-1}(|X|)\right]<\infty$, it follows that $\varphi^{-1}(|X|)<\infty$ a.s. Hence, we have by (3.1) that

$$
\begin{aligned}
\sum_{n=1}^{\infty} \frac{X^{2} I(|X| \leq \varphi(n))}{\varphi^{2}(n)} & =\sum_{n=1}^{\left\lceil\varphi^{-1}(|X|)\right\rceil+1} \frac{X^{2} I(|X| \leq \varphi(n))}{\varphi^{2}(n)}+\sum_{\left\lceil\varphi^{-1}(|X|)\right\rceil+2}^{\infty} \frac{X^{2} I(|X| \leq \varphi(n))}{\varphi^{2}(n)} \\
& \leq\left\lceil\varphi^{-1}(|X|)\right\rceil+1+\sum_{\left\lceil\varphi^{-1}(|X|)\right\rceil+2}^{\infty} \frac{X^{2}}{\varphi^{2}(n)} \\
& \leq \varphi^{-1}(|X|)+1+X^{2} \int_{\varphi^{-1}(|X|)}^{\infty} \frac{1}{\varphi^{2}(x)} d x \\
& \leq \varphi^{-1}(|X|)+1+a \varphi^{-1}(|X|)+b \\
& =(1+a) \varphi^{-1}(|X|)+1+b,
\end{aligned}
$$

which implies that

$$
E\left[\sum_{n=1}^{\infty} \frac{X^{2} I(|X| \leq \varphi(n))}{\varphi^{2}(n)}\right] \leq(1+a) E\left[\varphi^{-1}(|X|)\right]+1+b<\infty .
$$

Combining (4.3) and (4.4), we can see that

$$
\sum_{n=1}^{\infty} \frac{\operatorname{Var}\left(Y_{n}\right)}{\varphi^{2}(n)} \leq \sum_{n=1}^{\infty} \frac{E Y_{n}^{2}}{\varphi^{2}(n)}<\infty
$$


It follows by Lemma 2.1 that $\left\{Y_{n} / \varphi(n), n \geq 1\right\}$ is also a sequence of AANA random variables. Hence, we have by (4.5) and Lemma 2.2 that

$$
\sum_{n=1}^{\infty} \frac{Y_{n}-E Y_{n}}{\varphi(n)} \text { converges a.s., }
$$

which together with (4.2) implies that

$$
\sum_{n=1}^{\infty} \frac{X_{n}-E Y_{n}}{\varphi(n)} \text { converges a.s. }
$$

To complete the proof of (3.2), it suffices to show that

$$
\begin{aligned}
& \sum_{n=1}^{\infty} \frac{\varphi(n) P\left(X_{n}<-\varphi(n)\right)-\varphi(n) P\left(X_{n}>\varphi(n)\right)}{\varphi(n)} \\
& \quad=\sum_{n=1}^{\infty}\left[P\left(X_{n}<-\varphi(n)\right)-P\left(X_{n}>\varphi(n)\right)\right] \quad \text { converges. }
\end{aligned}
$$

But this follows from (4.1) immediately. This completes the proof of (3.2). Finally, $\frac{1}{f(n)} \sum_{i=1}^{n} \frac{X_{i}-m_{i}}{g(i)} \rightarrow 0$ a.s. as $n \rightarrow \infty$ follows from (4.1) and Kronecker's lemma immediately. The proof is complete.

Proof of Theorem 3.2 We use the same notations as those in Theorem 3.1. In the proof of Theorem 3.1, we have proved that

$$
\sum_{n=1}^{\infty} P\left(\left|X_{n}\right|>\varphi(n)\right) \leq C \sum_{n=1}^{\infty} P(|X|>\varphi(n))<\infty
$$

and

$$
\sum_{n=1}^{\infty} \frac{\operatorname{Var}\left(Y_{n}\right)}{\varphi^{2}(n)}<\infty
$$

Note that $\left\{Y_{n} / \varphi(n), n \geq 1\right\}$ is also a sequence of AANA random variables. By Lemma 2.3, we can see that in order to prove (3.3), we only need to show

$$
\sum_{n=1}^{\infty} \frac{E Y_{n}}{\varphi(n)} \text { converges. }
$$

Suppose that (i) holds, we have by Lemma 2.4 that

$$
\begin{aligned}
\sum_{n=1}^{\infty}\left|\frac{E Y_{n}}{\varphi(n)}\right| & \leq \sum_{n=1}^{\infty} \frac{1}{\varphi(n)}\left[E\left|X_{n}\right| I\left(\left|X_{n}\right| \leq \varphi(n)\right)+\varphi(n) P\left(\left|X_{n}\right|>\varphi(n)\right)\right] \\
& \leq C \sum_{n=1}^{\infty} \frac{1}{\varphi(n)} E|X| I(|X| \leq \varphi(n))+C \sum_{n=1}^{\infty} P(|X|>\varphi(n)) \\
& \leq C \sum_{n=1}^{\infty} \frac{1}{\varphi(n)} \sum_{j=1}^{n} E|X| I\left(j-1<\varphi^{-1}(|X|) \leq j\right)
\end{aligned}
$$




$$
\begin{aligned}
& \leq C \sum_{n=1}^{\infty} \frac{1}{\varphi(n)} \sum_{j=1}^{n} \varphi(j) P\left(j-1<\varphi^{-1}(|X|) \leq j\right) \\
& =C \sum_{j=1}^{\infty} \varphi(j) P\left(j-1<\varphi^{-1}(|X|) \leq j\right) \sum_{n=j}^{\infty} \frac{1}{\varphi(n)} \\
& \leq C \sum_{j=1}^{\infty} \varphi(j) P\left(j-1<\varphi^{-1}(|X|) \leq j\right)\left[\frac{1}{\varphi(j)}+\int_{j}^{\infty} \frac{1}{\varphi(x)} d x\right] \\
& \leq C \sum_{j=1}^{\infty} j P\left(j-1<\varphi^{-1}(|X|) \leq j\right) \\
& \leq C \sum_{n=1}^{\infty} P\left(\varphi^{-1}(|X|) \geq n\right) \\
& \leq C E\left[\varphi^{-1}(|X|)\right]<\infty,
\end{aligned}
$$

which implies (4.6).

Suppose that (ii) holds. Note that $E X_{i}=0$. We have by Lemma 2.4 again that

$$
\begin{aligned}
\sum_{n=1}^{\infty}\left|\frac{E Y_{n}}{\varphi(n)}\right| & \leq \sum_{n=1}^{\infty} \frac{1}{\varphi(n)}\left[\left|E X_{n} I\left(\left|X_{n}\right| \leq \varphi(n)\right)\right|+\varphi(n) P\left(\left|X_{n}\right|>\varphi(n)\right)\right] \\
& \leq \sum_{n=1}^{\infty} \frac{1}{\varphi(n)}\left[E\left|X_{n}\right| I\left(\left|X_{n}\right|>\varphi(n)\right)+\varphi(n) P\left(\left|X_{n}\right|>\varphi(n)\right)\right] \\
& \leq C \sum_{n=1}^{\infty} \frac{1}{\varphi(n)} E|X| I(|X|>\varphi(n))+C \sum_{n=1}^{\infty} P(|X|>\varphi(n)) \\
& \leq C \sum_{n=1}^{\infty} \frac{1}{\varphi(n)} \sum_{j=n}^{\infty} E|X| I\left(j<\varphi^{-1}(|X|) \leq j+1\right) \\
& \leq C \sum_{n=1}^{\infty} \frac{1}{\varphi(n)} \sum_{j=n}^{\infty} \varphi(j+1) P\left(j<\varphi^{-1}(|X|) \leq j+1\right) \\
& =C \sum_{j=1}^{\infty} \varphi(j+1) P\left(j<\varphi^{-1}(|X|) \leq j+1\right) \sum_{n=1}^{j} \frac{1}{\varphi(n)} \\
& \leq C \sum_{j=1}^{\infty} \varphi(j+1) P\left(j<\varphi^{-1}(|X|) \leq j+1\right)\left[\frac{1}{\varphi(1)}+\int_{1}^{j+1} \frac{1}{\varphi(x)} d x\right] \\
& \leq C \sum_{j=1}^{\infty}(j+1) P\left(j<\varphi^{-1}(|X|) \leq j+1\right) \\
& \leq C\left[\sum_{j=1}^{\infty} j P\left(j<\varphi^{-1}(|X|) \leq j+1\right)+\sum_{j=1}^{\infty} P\left(j<\varphi^{-1}(|X|) \leq j+1\right)\right] \\
& {\left.\left[\varphi^{-1}(|X|)\right]+1\right\}<\infty, } \\
&
\end{aligned}
$$

which implies (4.6). 
Thus, (4.6) holds both in case (i) and in case (ii). This completes the proof of the theorem.

Proof of Corollary 3.1 If $0<p<1$, then for an arbitrary real number $a$,

$$
\frac{1}{n^{1 / p}} \sum_{i=1}^{n} a \rightarrow 0 \quad \text { as } n \rightarrow \infty .
$$

Thus, in order to prove (3.4), we only need to show that

$$
\frac{1}{n^{1 / p}} \sum_{i=1}^{n} X_{i} \rightarrow 0 \quad \text { a.s. as } n \rightarrow \infty .
$$

If $1<p<2, a=E X_{1}$, without loss of generality, we assume that $E X_{1}=0$. Hence, for $0<p<2$ and $p \neq 1$, we only need to show (4.7) holds true.

Taking

$$
\begin{aligned}
& f(x)=x^{1 / p}, \quad 0<p<2, p \neq 1, x \in(0, \infty) ; \\
& g(x)=1, \quad x \in(0, \infty) ; \\
& \varphi(x)=f(x) g(x), \quad x \in(0, \infty), \varphi(0)=0,
\end{aligned}
$$

in Theorem 3.2, we can get the desired result (3.4) immediately.

For $p=1, f(x), g(x)$ and $\varphi(x)$ satisfy the conditions of Theorem 3.1, hence,

$$
\frac{1}{n} \sum_{i=1}^{n}\left(X_{i}-m_{i}\right) \rightarrow 0 \quad \text { a.s. as } n \rightarrow \infty
$$

where $m_{i}=E X_{i} I\left(\left|X_{i}\right| \leq \varphi(i)\right)=E X_{1} I\left(\left|X_{1}\right| \leq \varphi(i)\right)$. By the dominated convergence theorem, we can get that $m_{i} \rightarrow E X_{1}$, which implies that

$$
\frac{1}{n} \sum_{i=1}^{n} m_{i} \rightarrow E X_{1}
$$

Therefore, the desired result (3.4) follows from (4.8) and (4.9) immediately. This completes the proof of the corollary.

Proof of Theorem 3.3 We use the same notations as those in Theorem 3.1. By (i), we can see that

$$
\begin{aligned}
\sum_{n=1}^{\infty} P\left(X_{n} \neq X_{n} I\left(\left|X_{n}\right| \leq \varphi(n)\right)\right) & =\sum_{n=1}^{\infty} P\left(\left|X_{n}\right|>\varphi(n)\right) \\
& \leq C \sum_{n=1}^{\infty} P(|X|>\varphi(n))<\infty
\end{aligned}
$$

which together with the Borel-Cantelli lemma yields that

$$
P\left(X_{n} \neq X_{n} I\left(\left|X_{n}\right| \leq \varphi(n)\right), \text { i.o. }\right)=0 \text {. }
$$


Note that $\left\{Y_{n} / \varphi(n), n \geq 1\right\}$ is still a sequence of AANA random variables, and by (4.3)

$$
\begin{aligned}
\sum_{n=1}^{\infty} \frac{\operatorname{Var}\left(Y_{n}\right)}{\varphi^{2}(n)} & \leq \sum_{n=1}^{\infty} \frac{E Y_{n}^{2}}{\varphi^{2}(n)} \\
& \leq C \sum_{n=1}^{\infty} \frac{E X^{2} I(|X| \leq \varphi(n))}{\varphi^{2}(n)}+C \sum_{n=1}^{\infty} P(|X|>\varphi(n)) \\
& <\infty .
\end{aligned}
$$

We have by Lemma 2.2 that

$$
\begin{aligned}
& \sum_{n=1}^{\infty}\left[\frac{X_{n} I\left(\left|X_{n}\right| \leq \varphi(n)\right)-m_{n}}{\varphi(n)}-I\left(X_{n}<-\varphi(n)\right)+I\left(X_{n}>\varphi(n)\right)\right. \\
& \left.\quad+P\left(X_{n}<-\varphi(n)\right)-P\left(X_{n}>\varphi(n)\right)\right] \quad \text { converges a.s. }
\end{aligned}
$$

The fact $\sum_{n=1}^{\infty} P\left(\left|X_{n}\right|>\varphi(n)\right)<\infty$ and the monotone convergence theorem yield that

$$
\sum_{n=1}^{\infty}\left[I\left(\left|X_{n}\right|>\varphi(n)\right)+P\left(\left|X_{n}\right|>\varphi(n)\right)\right] \text { converges a.s. }
$$

Combining (4.11) and (4.12), we can get that

$$
\sum_{n=1}^{\infty} \frac{X_{n} I\left(\left|X_{n}\right| \leq \varphi(n)\right)-m_{n}}{\varphi(n)} \quad \text { converges a.s. }
$$

The desired result (3.2) follows from (4.10) and (4.13) immediately. This completes the proof of the theorem.

Proof of Theorem 3.4 By (i), we can easily get

$$
\sum_{n=1}^{\infty} P\left(\left|X_{n}\right|>\varphi(n)\right) \leq C \sum_{n=1}^{\infty} P(|X|>\varphi(n)) \leq C \sum_{n=1}^{\infty} \frac{1}{\varphi(n)} E|X| I(|X|>\varphi(n))<\infty
$$

Applying Theorem 3.3, we can get (3.2) immediately. In order to prove (3.5), it suffices to show that

$$
\sum_{n=1}^{\infty} \frac{E X_{n} I\left(\left|X_{n}\right|>\varphi(n)\right)}{\varphi(n)} \quad \text { converges. }
$$

By (i) again and Lemma 2.4, we have

$$
\begin{aligned}
\sum_{n=1}^{\infty}\left|\frac{E X_{n} I\left(\left|X_{n}\right|>\varphi(n)\right)}{\varphi(n)}\right| & \leq \sum_{n=1}^{\infty} \frac{E\left|X_{n}\right| I\left(\left|X_{n}\right|>\varphi(n)\right)}{\varphi(n)} \\
& \leq C \sum_{n=1}^{\infty} \frac{E|X| I(|X|>\varphi(n))}{\varphi(n)} \\
& <\infty,
\end{aligned}
$$


which implies (4.15). The desired result (3.5) follows from (3.2) and (4.15) immediately. The proof is complete.

\section{Competing interests}

The author declares that they have no competing interests.

\section{Author's contributions}

The author read and approved the final manuscript.

\section{Acknowledgements}

The author are most grateful to the editor Andrei Volodin and an anonymous referee for careful reading of the manuscript and valuable suggestions which helped in improving an earlier version of this paper. This work was supported by the Project of the Feature Specialty of China (TS11496) and the Scientific Research Projects of Fuyang Teachers College (2009FSKJ09)

Received: 28 August 2012 Accepted: 13 December 2012 Published: 3 January 2013

\section{References}

1. Jajte, R: On the strong law of large numbers. Ann. Probab. 31, 409-412 (2003)

2. Jing, BY, Liang, HY: Strong limit theorems for weighted sums of negatively associated random variables. J. Theor. Probab. 21, 890-909 (2008)

3. Meng, YJ, Lin, ZY: Strong laws of large numbers for $\tilde{\rho}$-mixing random variables. J. Math. Anal. Appl. 365, 711-717 (2010)

4. Wang, ZZ: On strong law of large numbers for dependent random variables. J. Inequal. Appl. 2011, Article ID 279754 (2011)

5. Sung, $\mathrm{SH}$ : On the strong law of large numbers for weighted sums of random variables. Comput. Math. Appl. 62 4277-4287 (2011)

6. Joag-Dev, K, Proschan, F: Negative association of random variables with applications. Ann. Stat. 11(1), 286-295 (1983)

7. Chandra, TK, Ghosal, S: Extensions of the strong law of large numbers of Marcinkiewicz and Zygmund for dependent variables. Acta Math. Hung. 71(4), 327-336 (1996a)

8. Chandra, TK, Ghosal, S: The strong law of large numbers for weighted averages under dependence assumptions. J. Theor. Probab. 9(3), 797-809 (1996b)

9. Wang, YB, Yan, JG, Cheng, FY: The strong law of large numbers and the iterated logarithm for product sums of NA and AANA random variables. Southeast Asian Bull. Math. 27, 369-384 (2003)

10. Ko, MH, Kim, TS, Lin, ZY: The Hájek-Rényi inequality for the AANA random variables and its applications. Taiwan. J. Math. 9(1), 111-122 (2005)

11. Yuan, $D M, A n, J$ : Rosenthal type inequalities for asymptotically almost negatively associated random variables and applications. Sci. China Ser. A 52(9), 1887-1904 (2009)

12. Yuan, DM, Wu, XS: Limiting behavior of the maximum of the partial sum for asymptotically negatively associated random variables under residual Cesàro alpha-integrability assumption. J. Stat. Plan. Inference 140, 2395-2402 (2010)

13. Wang, XJ, Hu, SH, Yang, WZ: Convergence properties for asymptotically almost negatively associated sequence. Discrete Dyn. Nat. Soc. 2010, Article ID 218380 (2010)

14. Yuan, DM, An, J: Laws of large numbers for Cesàro alpha-integrable random variables under dependence condition AANA or AQSI. Acta Math. Sin. Engl. Ser. 28(6), 1103-1118 (2012)

15. Wang, XJ, Hu, SH, Yang, WZ, Wang, XH: On complete convergence of weighted sums for arrays of rowwise asymptotically almost negatively associated random variables. Abstr. Appl. Anal. 2012, Article ID 315138 (2012)

16. Yang, WZ, Wang, XJ, Ling, NX, Hu, SH: On complete convergence of moving average process for AANA sequence. Discrete Dyn. Nat. Soc. 2012, Article ID 863931 (2012)

17. Wang, XJ, Hu, SH, Li, XQ, Yang, WZ: Maximal inequalities and strong law of large numbers for AANA sequences. Commun. Korean Math. Soc. 26(1), 151-161 (2011)

doi:10.1186/1029-242X-2013-4

Cite this article as: Tang: Some strong laws of large numbers for weighted sums of asymptotically almost negatively associated random variables. Journal of Inequalities and Applications 2013 2013:4. 